\title{
Research on the micromotion characteristics of a chaff cloud
}

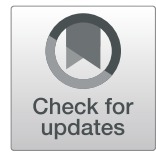

\author{
Ran LiD, Xinhong Hao* and Ping Li
}

\begin{abstract}
This paper reports the investigation on the micromotion characteristics of chaff dipoles and a chaff cloud. Micromotion models of chaff dipoles and steady-state chaff clouds are discussed. Fourier transform method and time-frequency method are used to study the micromotion features of chaff dipoles, in the energy domain and time-frequency domain, respectively. Based on the characteristic parameters in these two domains, the simulation results show that there are micro-Doppler frequency components in the echo signals from chaff dipoles, and these frequency components vary periodically. Then, the micro-Doppler frequencies of chaff cloud are obtained based on the method of multipoint synthesis. Finally, the experimental results of chaff dipoles dispersion verify the micromotion characteristics of chaff dipoles and chaff cloud obtained by using the empirical mode decomposition method and the short-time Fourier transform method.
\end{abstract}

Keywords: Micro-Doppler, Chaff cloud, Empirical mode decomposition, Time-frequency analysis

\section{Introduction}

Chaff cloud, which consists of a large number of chaff dipoles and possesses the reflection characteristics of large bandwidth, high density, and high power, has played an important role in electronic warfare. Releasing chaff cloud by chaff bombs is an effective deception interference measure for certain types of radar systems. Therefore, the technique of classifying chaff cloud and targets (such as missiles and aircrafts) for radar systems faces critical challenge [1-3]. It is well known that radar targets usually have micromotion characteristics that can be used to classify different targets [4]. Thus, we could study the micromotion characteristics of the chaff cloud to make a foundation for chaff cloud classification.

First of all, the micromotion models of radar targets are generally built in order to obtain the micromotion characteristics. Chen et al. [4] proposed two basic micromotion models, including the rotation and the oscillation. Then on that basis, Deng et al. [5], Li et al. [6], and Persico et al. $[7,8]$ proposed the micromotion models of

\footnotetext{
* Correspondence: haoxinhong@bit.edu.cn

Science and Technology on Electromechanical Dynamic Control Laboratory, School of Mechatronical Engineering, Beijing Institute of Technology, Beijing 100081, China
}

several kinds of radar targets, such as aircrafts, ships, and missiles, respectively.

Thus, the different micro-Doppler frequencies of different targets could be extracted from these above models. The time-frequency analysis [9] including the short-time Fourier transform (STFT) and the Wigner-Ville distribution is the simpler method to extract micro-Doppler frequency, and the adaptive chirplet representation [10] is a senior extraction algorithm. Subsequently, Li et al. [11] proposed an orthogonal matching pursuit algorithm to extract the micro-Doppler frequencies.

Then, the different classification technologies based on the micro-Doppler frequencies have been developed. Clemente et al. [12], Bai et al. [13], and Kim et al. [14] proposed the pseudo-Zernike moment method, the empirical mode decomposition (EMD), and the support vector machine (SVM), respectively.

However, the scholars did not study the micromotion characteristics of chaff clouds. The previous research on the characteristics of chaff clouds has involved three aspects: the aerodynamic characteristics [15], the statistical characteristics $[16,17]$, and the echo characteristics [18-20]. Brunk et al. [15] proposed a spiral descent motion model of a single chaff dipole by a lot of experiments. James et al. [16] modified the operational hydrometeor classification algorithm (HCA) for the WSR-88D weather radar to include a chaff class that can 
be used as an input to a chaff detection algorithm. The research on the echo characteristics of chaff clouds has involved three aspects: the Doppler, the pulse-pulse correlation, and the depolarization research [18-20].

In view of the above analysis, the aim of this paper is to demonstrate that chaff dipoles and chaff clouds have micro-Doppler effects that can be used to carry out preliminary theoretical research for identifying targets and chaff clouds. After the spiral motion model of chaff dipoles is studied and extended through dynamic analysis, a new micromotion model of chaff dipoles is proposed, which is defined in terms of precession micromotion. Then, the micro-Doppler frequencies of chaff dipoles are obtained. Furthermore, the micro-Doppler frequencies of a chaff cloud are obtained based on the method of chaff dipoles multipoint synthesis. The remainder of this paper is structured as follows: in Section 2, the precession model is given by the dynamic characteristics of the chaff dipoles. In Section 3, based on the theory of the micromotion characteristics of a single chaff dipole, the micro-Doppler frequency features of a chaff cloud are quantified by the multipoint synthesis method. A numerical solution of the micro-Doppler frequency of a chaff cloud is given, and the timefrequency spectrum and the energy spectrum of the chaff cloud are obtained. In Section 4, the experimental results of a chaff dipole dispersion experiment verify the accuracy of the above conclusions in Section 2 and Section 3. Finally, the conclusions are provided in Section 5.

\section{Micromotion theoretical model}

When tens of thousands of chaff dipoles with a small mass are thrown from an aircraft, a chaff cloud forms over time in the air. A chaff dipole has a certain initial precessional angular velocity as it is thrown. If the processing error of a chaff dipole is not considered so that its centroid is assumed to coincide with its shape center completely, gravity and atmospheric resistance do not produce a precession moment. Typically, the chaff dipoles move at a constant angular velocity. Under this assumption, the motion of a chaff cloud in a radar coordinate system is approximately the uniform linear motion, defined by a chaff cloud steady-state model. Compared with the traditional micromotion of aircraft propellers [4], the essential difference in terms of micromotion feature is that the coordinate values of a chaff dipole vary with respect to each time-varying center, while a propeller rotates around a fixed center axis. Therefore, we need to analyze the micromotion characteristics of chaff dipoles and a chaff cloud in terms of coordinates, distances, elevations, and azimuths.

The steady-state model of a chaff cloud is generally a solid spherical or ellipsoidal model, in which the chaff cloud moves in a uniform straight line along a certain direction. The chaff dipoles are distributed randomly on a spherical surface or inside the spherical model. The spacing of the chaff dipoles should be larger than $2 \lambda$, so there is no coupling phenomenon [21]. The length of a single chaff dipole is $l_{c}=\lambda / 2$ according to the induction scattering theory [22]. The diameter $d$ of a chaff dipole cross-sectional area is

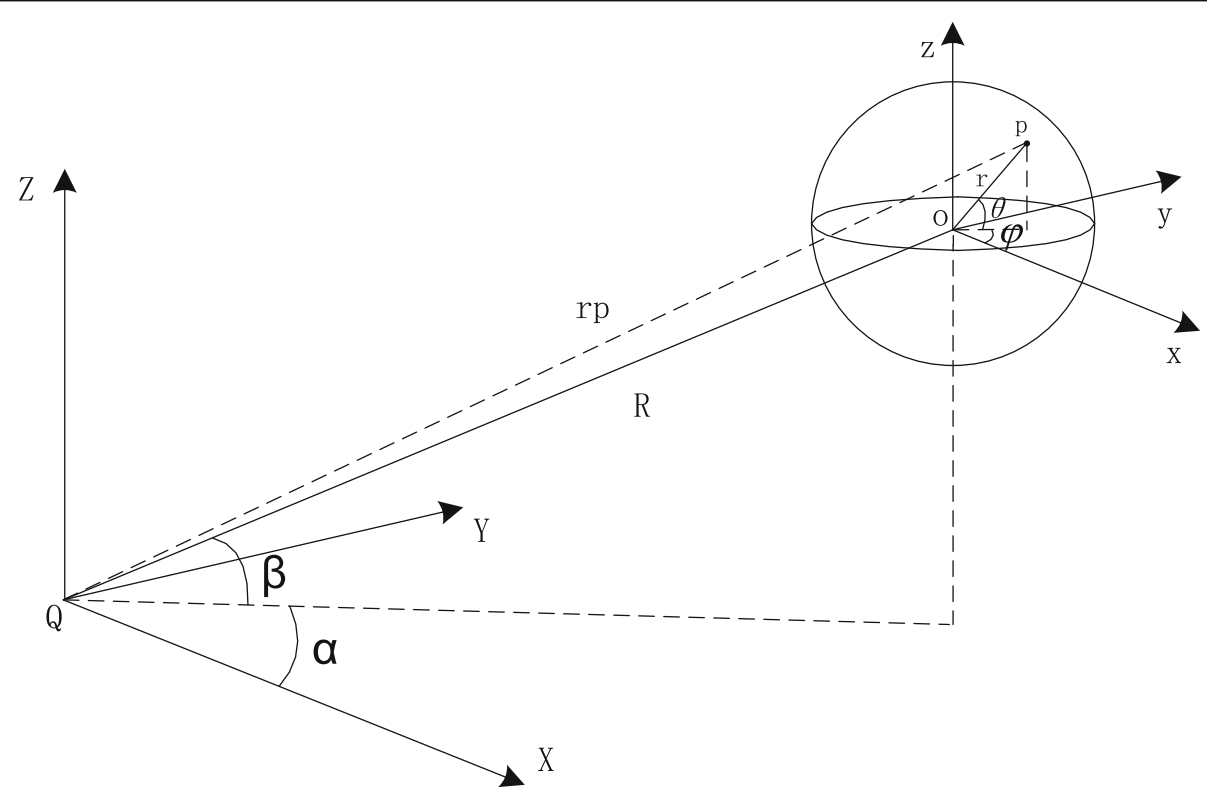

Fig. 1 Position model of a single chaff dipole at point $P$ 
$d<<\lambda / 2$. The reference coordinate system is constructed by taking the center of the chaff cloud as the origin of the coordinate. In the reference coordinate system, the elevation angle and the azimuth angle of a single chaff dipole at the point $P$ in the chaff cloud are defined as $\theta$ and $\phi$, respectively. Figure 1 shows that $r$ is the distance from the point $P$ to the origin of the reference coordinate system. The reference position $P$ can be expressed as $\left[x_{p}, y_{p}, z_{p}\right]$ in the reference coordinate system. After that, the radar coordinate system is constructed by taking the center of the radar system as the origin of the coordinate. In the radar coordinate system, the elevation angle and the azimuth angle of the chaff cloud are defined as $\beta$ and $\alpha$, respectively. Then, the reference position $P$ can be expressed as $\left[X_{p}, Y_{p}\right.$, $\left.Z_{p}\right]$ in the radar coordinate.

According to the Euler coordinate transformation formula [4], the position of a single chaff dipole in the radar coordinate system can be obtained as $\left[X_{p}, Y_{p}, Z_{p}\right]=\mathfrak{R}_{\text {init }}$ - $\left[x_{p}, y_{p}, z_{p}\right]$, where $\mathfrak{R}_{\text {init }}$ is the Euler coordinate transformation matrix [4], which is the first step in quantifying the precession characteristics of a single chaff dipole relative to the radar.

The radar echo modulation caused by precession is a special modulation mechanism of the chaff dipoles because different echo modulations are produced by the target (e.g., an aircraft or a ship) due to the different states of motion. Figure 2 shows that the precessional motion can be considered as a combination of conical motion (Fig. 2a) and spin motion (Fig. 2b).

As shown in Fig. 1, the distance between a single precessing chaff dipole relative to the radar is as follows:

$$
\overrightarrow{O p}=\vec{r}+\Re_{\text {precession }} \overrightarrow{r_{d}}
$$

Thus, we can obtain the distance modulus at time $t$ as $r_{p}(t)=\left\|\overrightarrow{R\left(t_{1}\right)}+\overrightarrow{r\left(t_{2}\right)}+\Re_{\text {precession }} \overrightarrow{r_{d}(t)}\right\|\left(t=t_{1}+t_{2}\right)$.

The parameters of Formula (1) are as follows:

1. $\overrightarrow{r\left(t_{2}\right)}$-the coordinate vector of the point $P$ in the reference coordinate system.

2. $\overrightarrow{r_{d}(t)}$-the precession radius of a single chaff dipole.

3. precession - the precession matrix of a single chaff dipole in the reference coordinate system.

4. $\overrightarrow{R\left(t_{1}\right)}$-the vector of the chaff cloud center in the radar coordinate system.

5. $t_{1}$-the time delay of the echo from the chaff cloud center point.

6. $t_{2}$-the time delay from the chaff cloud center point to the reference point $P$.

We suppose that the radar transmits a singlefrequency continuous-wave $(\mathrm{CW})$ signal with a carrier frequency $f_{c}$ :

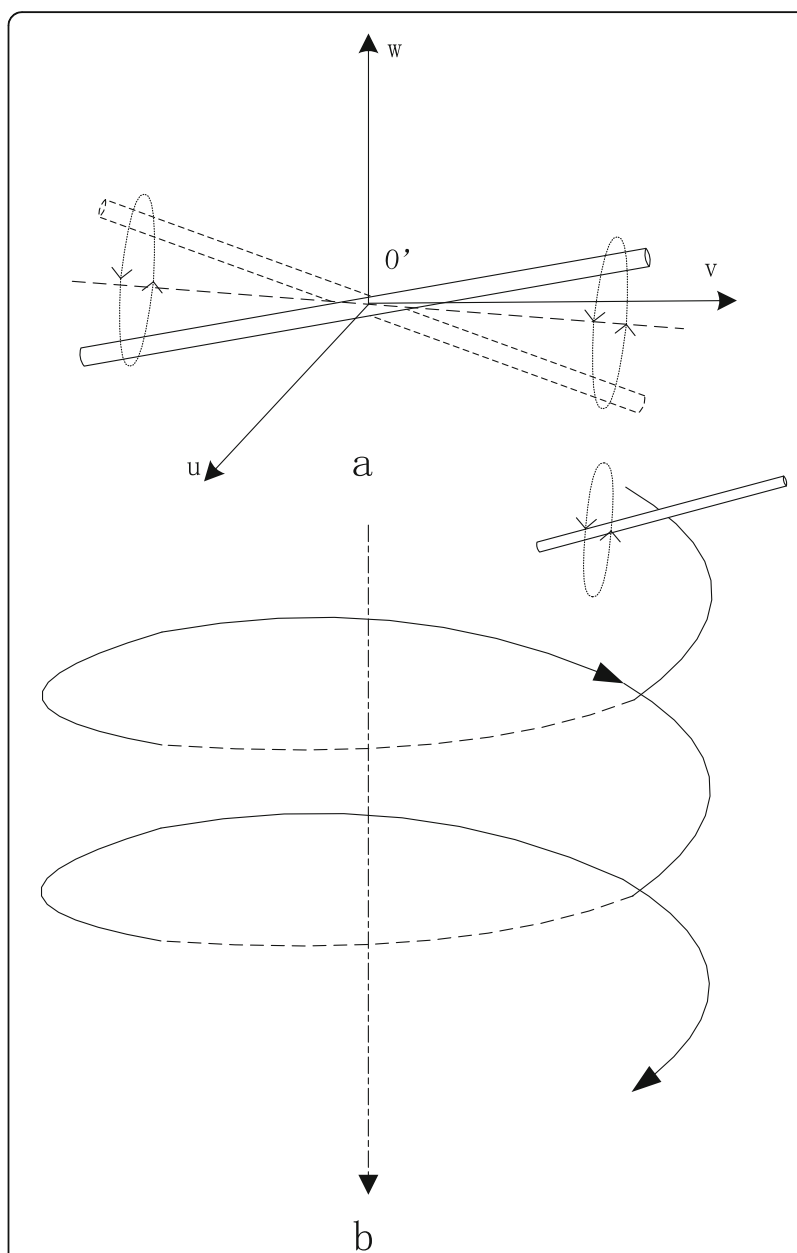

Fig. 2 Progressive model of a single chaff dipole precession motion: $\mathbf{a}$ the conical motion of a chaff dipole and $\mathbf{b}$ a falling chaff dipole at a constant angular velocity

$$
p(t)=\exp \left(j 2 \pi f_{c} t\right)
$$

After that, the echo signal of the scattering point $P$ is ${ }^{1}$ :

$$
s(t)=\sigma \exp \left\{j 2 \pi f_{c}\left[t-\frac{2 r_{d}(t)}{c}\right]\right\}
$$

where $r_{d}(t)$ is the value of the real vector $\overrightarrow{r_{d}(t)}$. A baseband transformation of $s(t)$ results in:

$$
s_{b}(t)=\sigma \exp \left\{j 2 \pi f_{c} \frac{2 r_{d}(t)}{c}\right\}=\sigma \exp \{j \phi(t)\}
$$

The parameters of Formula (4) are as follows:

1. $\phi(t)$-the phase term, $\phi(t)=2 \pi f_{c} \cdot\left(2 r_{d}(t) / c\right)$.

2. $\sigma$-the radar cross section (RCS) of the chaff dipole.

3. $c$-the velocity of light.

${ }^{1}$ There is a radar LOS (line of sight) for the scattering chaff cloud, so the value of the LOS angle coefficient of Formula (4) is 1 , and 0 otherwise [7]. 


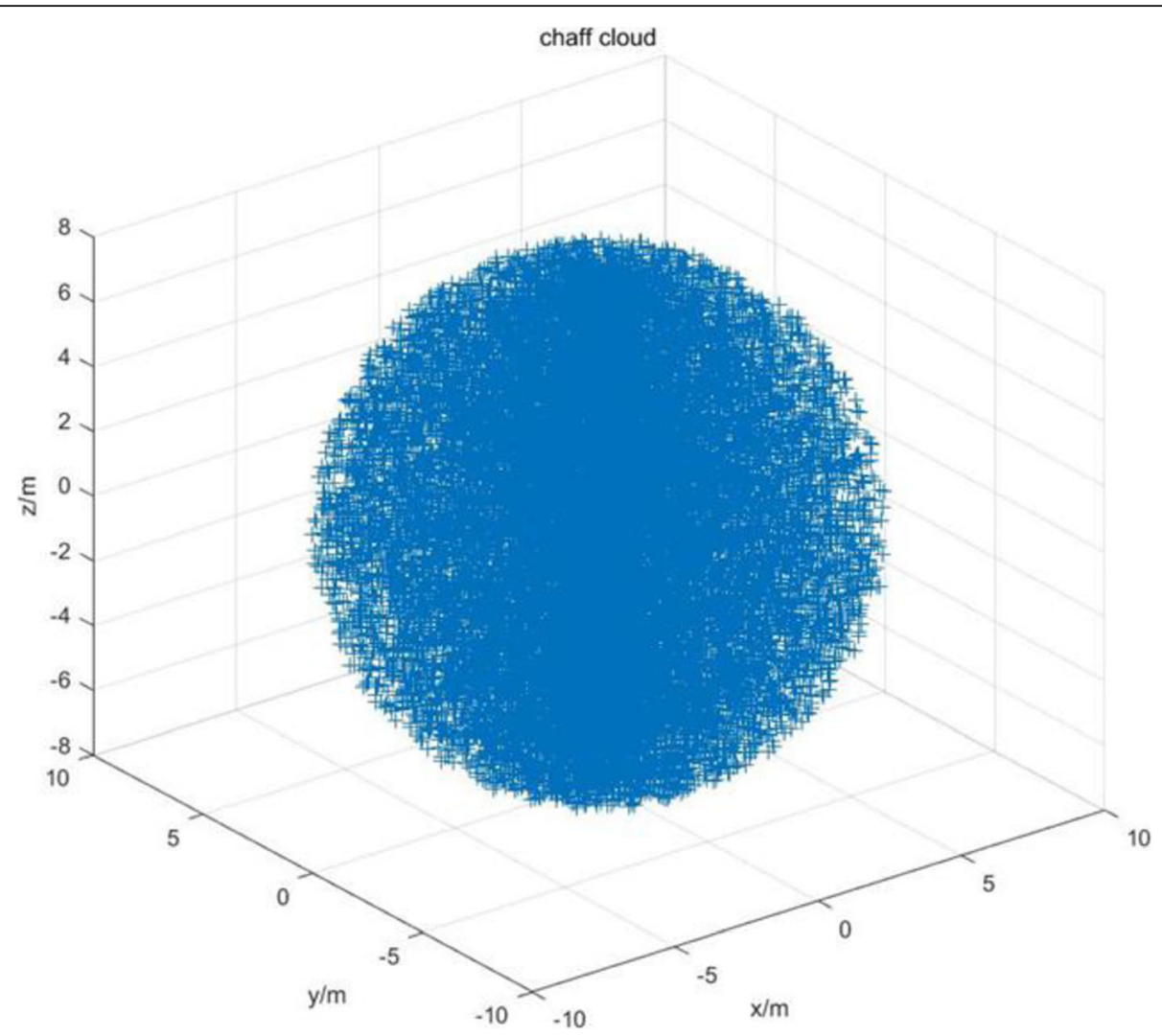

Fig. 3 A solid spherical steady-state model of the chaff cloud

The Doppler frequency of the echo signal can be obtained by deriving the phase term with respect to time $t$ :

$$
\begin{gathered}
f_{d}=\frac{1}{2 \pi} \frac{d \phi(t)}{d t} \\
=\frac{2 f_{c}}{c}\left[\frac{d\left(\Re_{\text {precession } \left.\overline{r_{d}(t)}\right)}\right.}{d t}\right]^{T} \vec{n}
\end{gathered}
$$

where the unit vector of $\overrightarrow{Q p}$ is $\vec{n}=(\overrightarrow{R(t)}+\overrightarrow{r(t)}$ $\left.+\Re_{\text {precession }} \overrightarrow{r(t)}\right) /\left(\left\|\overrightarrow{R(t)}+\overrightarrow{r(t)}+\Re_{\text {precession }} \overrightarrow{r(t)}\right\|\right)$, which is the coordinate vector of the point $O$ in the radar coordinate system in Fig. 1.

When the chaff dipole is in the far field of the radar, $\|\overrightarrow{R(t)}+\overrightarrow{r(t)}\|>>\left\|\Re_{\text {precession }} \overrightarrow{r(t)}\right\|$; therefore, the unit vector of the line of sight of the radar is simplified to $\vec{n} \approx(\overrightarrow{R(t)}+\overrightarrow{r(t)}) /\|\overrightarrow{R(t)}+\overrightarrow{r(t)}\|$. Then, the micro-Doppler frequency caused by the chaff dipole precession can be expressed as follows:

$$
f_{\text {micro- } D}=\frac{2 f_{c}}{c}\left[\frac{d\left(\Re_{\text {precession }} \overrightarrow{r_{d}(t)}\right)}{d t}\right]^{T} \vec{n}
$$

Furthermore, the precession matrix $\boldsymbol{R}_{\text {precession }}$ can be expressed as a conical motion matrix $\mathfrak{R}_{\text {con }}$ multiplied by a spin motion matrix $\Re_{\text {spin }}$.

$$
\boldsymbol{R}_{\text {precession }}=\boldsymbol{R}_{\text {con }} \cdot \mathfrak{R}_{\text {spin }}
$$

According to the Rodrigues formula [14], the conical motion matrix $\mathfrak{R}_{\text {con }}$ can be described as:

$$
\begin{aligned}
\Re_{\text {con }}= & I+\hat{\omega}_{\text {con }} \sin \left(\Omega_{\text {con }} t\right) \\
& +\hat{\omega}_{\text {con }}^{2}\left[1-\cos \left(\Omega_{\text {con }} t\right)\right]
\end{aligned}
$$

Here,

1. $\Omega_{\text {con }}$-the angular velocity of the conical motion.

2. $\hat{\omega}_{\text {con }}-$ the oblique symmetric matrix of the conical motion.

$$
\hat{\omega}_{\operatorname{con}}=\left[\begin{array}{ccc}
0 & -\sin \theta & \sin \phi \cos \theta \\
\sin \theta & 0 & -\cos \phi \cos \theta \\
-\sin \phi \cos \theta & \cos \phi \cos \theta & 0
\end{array}\right]
$$

Similarly, the spin matrix $\mathfrak{R}_{\text {spin }}$ can be expressed as: 


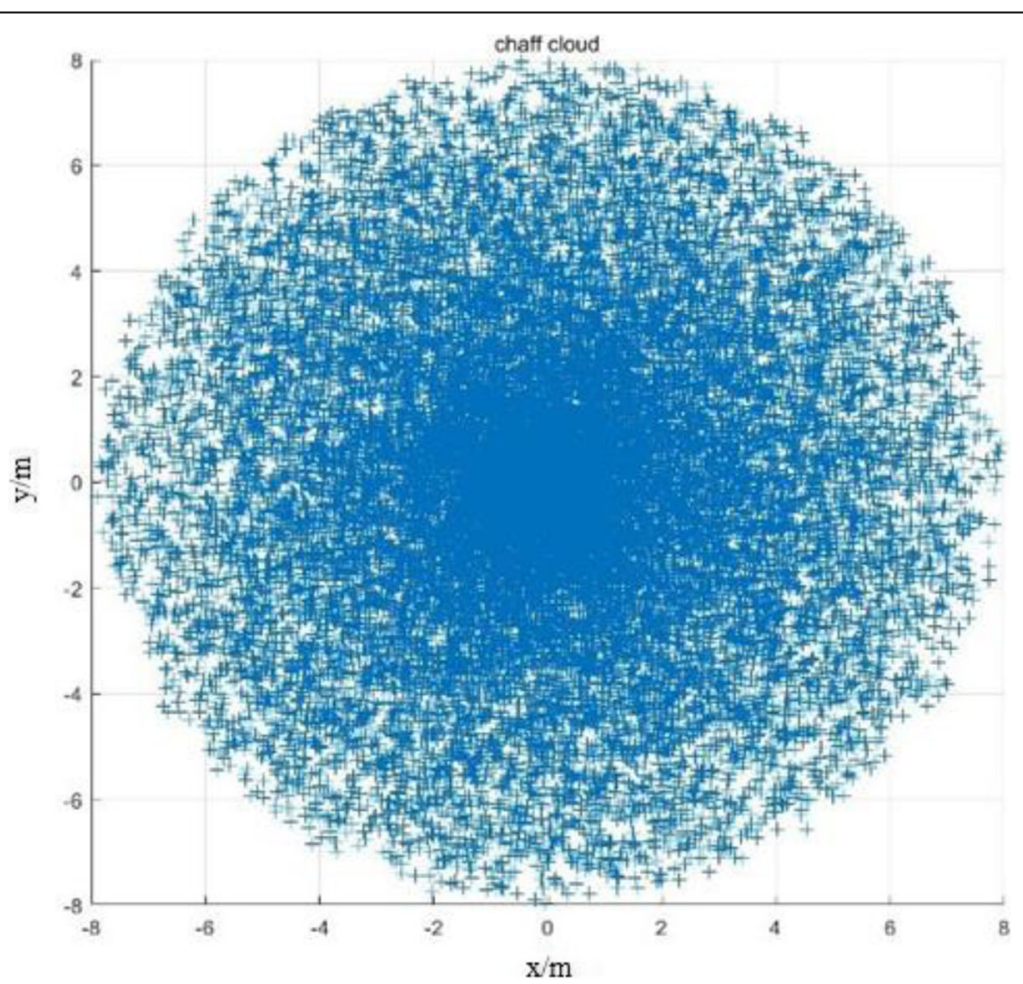

a

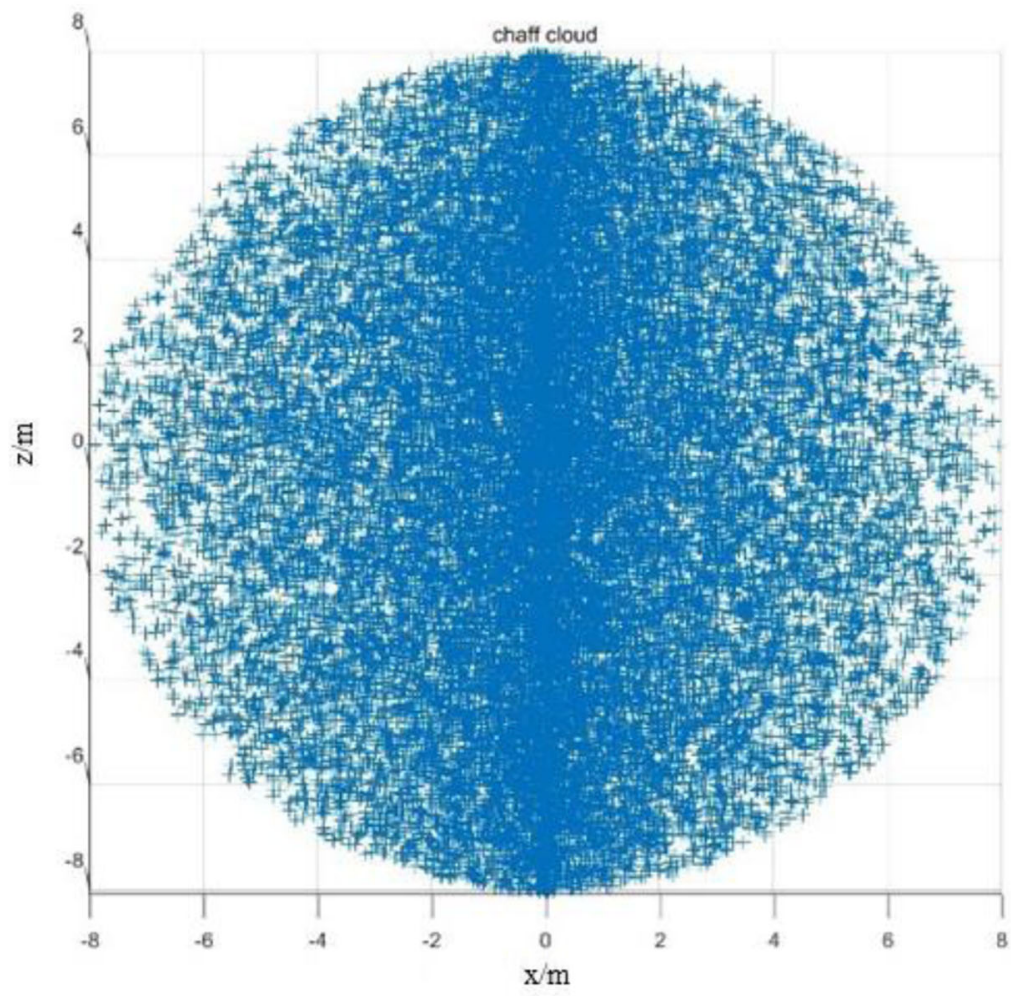

Fig. 4 The $x-y$ and $x-z$ cross sections of the chaff cloud: a the $x-y$ cross section, $\mathbf{b}$ the $x-z$ cross section 

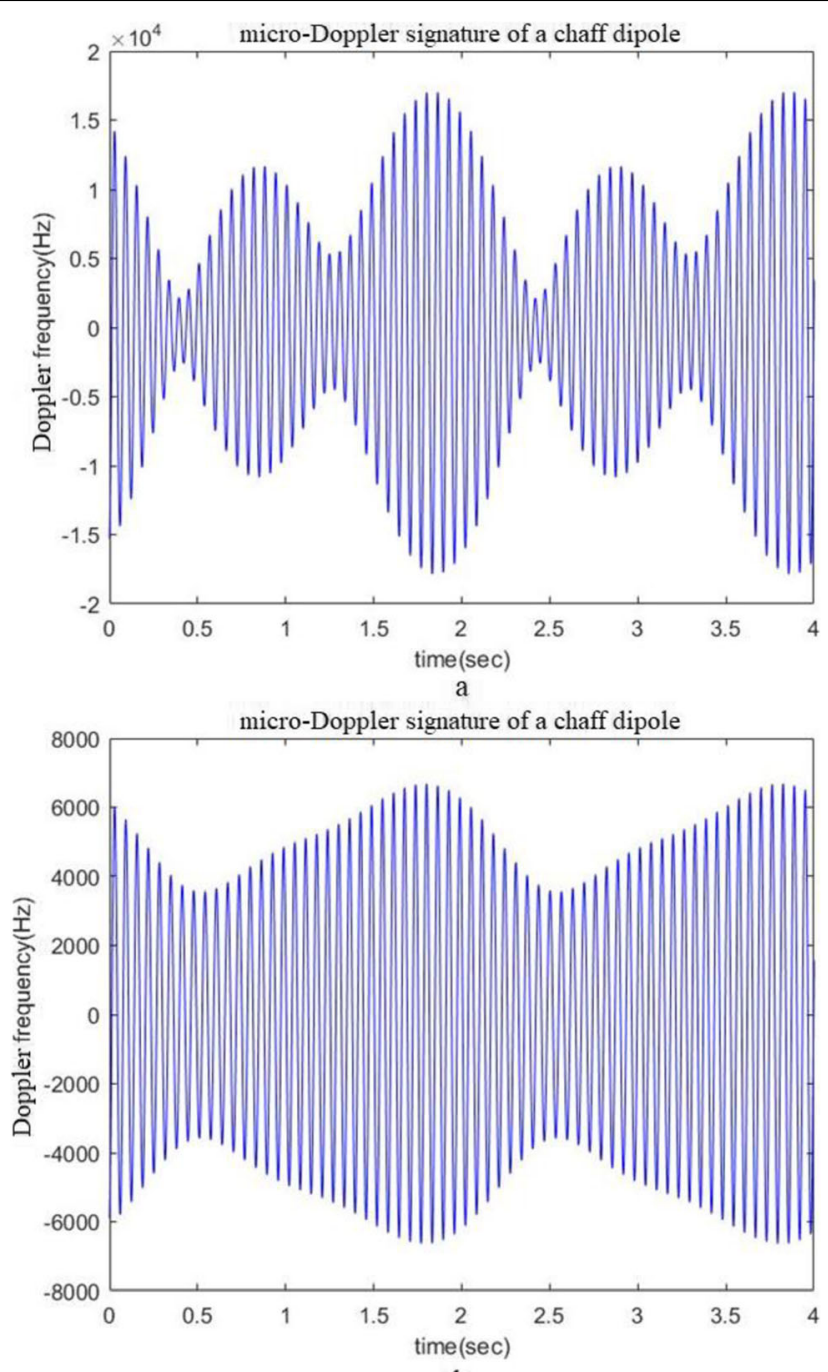

$\mathrm{b}$

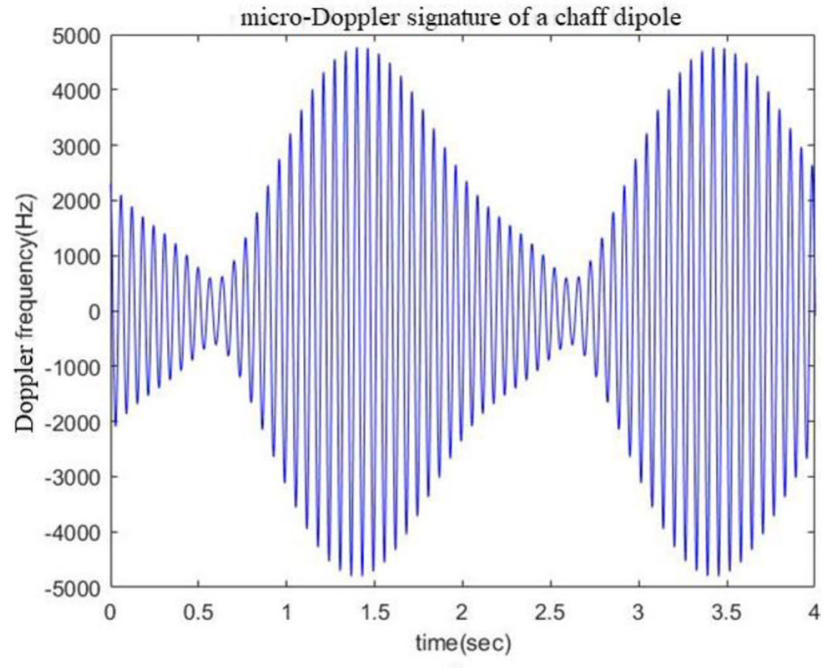

Fig. 5 (See legend on next page.) 
(See figure on previous page.)

Fig. 5 Micro-Doppler frequency of single chaff dipoles with different elevation angles (in the reference coordinates): a $\theta=15^{\circ}, \varphi=30^{\circ}, d_{r}=722 m$, b $\theta=45^{\circ}, \varphi=30^{\circ}, d_{r}=722 \mathrm{~m}$, and $\mathbf{c} \theta=75^{\circ}, \varphi=30^{\circ}, d_{r}=722 \mathrm{~m}$

$$
\begin{aligned}
\boldsymbol{R}_{\text {spin }}= & I+\hat{\omega}_{\text {spin }} \sin \left(\Omega_{\text {spin }} t\right) \\
& +\hat{\omega}_{\text {spin }}^{2}\left[1-\cos \left(\Omega_{\text {spin }} t\right)\right]
\end{aligned}
$$

Here,

1. $\Omega_{\text {spin }}$-the angular velocity of the spin motion.

2. $\hat{\omega}_{\text {spin }}$-the oblique symmetric matrix of the spin motion.

$$
\hat{\omega}_{\text {spin }}=\left[\begin{array}{ccc}
0 & -\omega_{z}^{\prime} & \omega_{y}^{\prime} \\
\omega_{z,}^{\prime} & 0 & -\omega_{x}^{\prime} \\
-\omega_{y}^{\prime} & \omega_{x}^{\prime} & 0
\end{array}\right]
$$

Here,

1. $T_{c}$-the spin axis, $T_{c}=\left(x_{c}, y_{c}, z_{c}\right)$.

2. $\omega_{c}$-the spin frequency.

Thus, $\omega_{x}^{\prime}=2 \pi \omega_{c} x_{c}, \omega_{y}^{\prime}=2 \pi \omega_{c} y_{c}$, and $\omega_{z}^{\prime}=2 \pi \omega_{c} z_{c}$.

Taking a single chaff dipole as a scattering point, the reference coordinate system will be moved to a new position in the radar coordinate system according to $\boldsymbol{R}_{\text {init }} \cdot \overrightarrow{r_{d}(t)}$. Referring to the radar coordinate system $(X, Y, Z)$, the scattering point $P$ will move from its initial position to a new position $\overrightarrow{r_{p}(t)}=\Re_{\text {precession }}$ $\cdot \mathfrak{R}_{\text {init }} \cdot \overrightarrow{r_{d}(t)}$ at time $t$. The micro-Doppler modulation of the scattering point $P$ due to precession can be expressed as follows:

$$
\begin{array}{r}
f_{\text {micro-D }}=\frac{2 f_{c}}{c}\left[\frac{d\left(\boldsymbol{R}_{\text {precession }} \cdot \boldsymbol{R}_{\text {init }} \cdot \overrightarrow{r_{d}(t)}\right)}{d t}\right]^{T} \overrightarrow{n^{\prime}} \\
=\frac{2 f_{c}}{c}\left[\left(\frac{d \boldsymbol{R}_{\text {con }}}{d t} \cdot \boldsymbol{R}_{\text {spin }}+\frac{d \boldsymbol{R}_{\text {spin }}}{d t} \cdot \boldsymbol{R}_{\text {con }}\right)\right. \\
\left.\cdot \Re_{\text {init }} \cdot \overrightarrow{r_{d}(t)}\right]^{T} \overrightarrow{n^{\prime}}
\end{array}
$$

\section{Simulation discussion and results}

Based on the above theoretical derivation, the micromotion characteristics of a chaff cloud are further analyzed. It is assumed that all the chaff dipoles in the chaff cloud are the same type (the materials, lengths, and diameters are the same). When the chaff cloud is stable in the radar coordinate system (where the chaff cloud motion approximately obeys the uniform linear motion), all the chaff dipoles can be considered to characterize the precessional motion at the same constant angular velocity. Figure 3 shows that a solid spherical chaff cloud model is constructed. The radius of the chaff cloud is $8 \mathrm{~m}$, and the center point of the chaff cloud is $(100,500,500)$ in the radar coordinate system. Then, the chaff cloud drops uniformly along the $Z$-axis of the radar coordinate system (i.e., the relative velocity of the movement between the radar and the chaff cloud in the radial direction is 0). Furthermore, the Euler transformation angle of the reference coordinate system is set to $(\pi / 6, \pi / 6, \pi / 4)$.

Finally, there are 25,920 chaff dipoles randomly distributed in the cloud model. The length of a single chaff dipole is $0.25 \mathrm{~cm}$, and the cross-sectional diameter $d$ of a chaff dipole is $d=25 \mu \mathrm{m}$. Since it is assumed that the radar transmitting wavelength is 0.5 $\mathrm{cm}$, the spacing of each chaff dipole $d_{c}$ satisfies the formula $d_{c} \geq 1 \mathrm{~cm}$, where $d_{c} \geq 2 \lambda$ satisfies the uncoupled condition. Figure 4 shows the $x-y$ and $x-z$ cross-sections of the chaff cloud.

According to the theory of the precession model of the chaff dipoles and assuming a conical angular velocity $\Omega_{\text {con }}=12 \mathrm{rad} / \mathrm{s}$ and a spin angular velocity $\Omega_{\text {spin }}=$ $3 \mathrm{rad} / \mathrm{s}$, we introduce $\Omega_{\text {con }}$ and $\Omega_{\text {spin }}$ into Formulas (8) and (10). Based on the method of multipoint synthesis (where the real target echo is obtained by superimposing the corresponding signals of each scattering point when the relative positions, scattering intensities and phases of the corresponding echo signals are different), the micro-Doppler frequency of a single chaff dipole in the chaff cloud is calculated and simulated based on the short-time Fourier transform, as shown from Figs. 5, 6 , and 7 .

Figure $5 \mathrm{a}-\mathrm{c}$ shows the three scattering points (the chaff dipoles at three points a, b, and c) selected randomly in the chaff cloud have the same distance $d_{r}$ (in the radar coordinate system), the same azimuth $\phi$, and different elevations $\theta$ (in the reference coordinate system). In contrast, from Fig. $5 \mathrm{a}-\mathrm{c}$, we can see that the chaff dipoles produce micro-Doppler frequencies with different bandwidths at different times and that the absolute range of the bandwidth is 4 to $20 \mathrm{kHz}$ through Formula (12). All the periods of the envelopes of the transient bandwidth of the microDoppler frequency in different situations are consistent with the angular frequency of the spin motion, $f_{\text {envelope }}=0.494 \mathrm{~Hz}$. It is worth noting that since the 


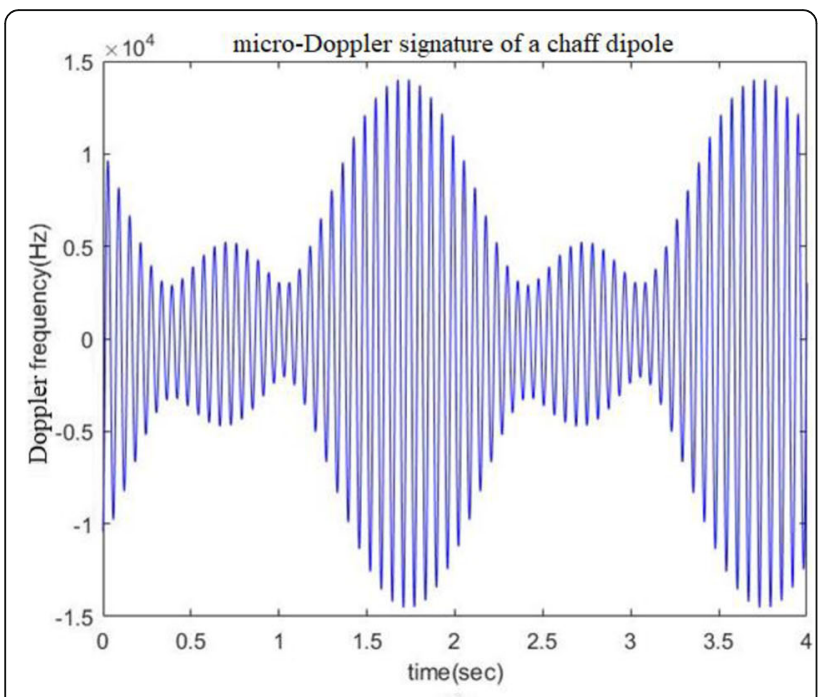

a

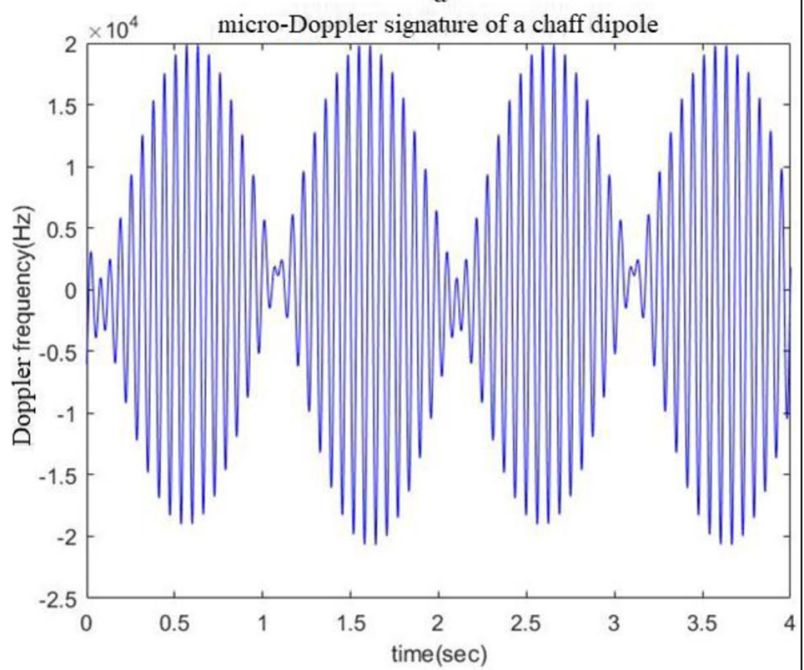

$\mathrm{b}$

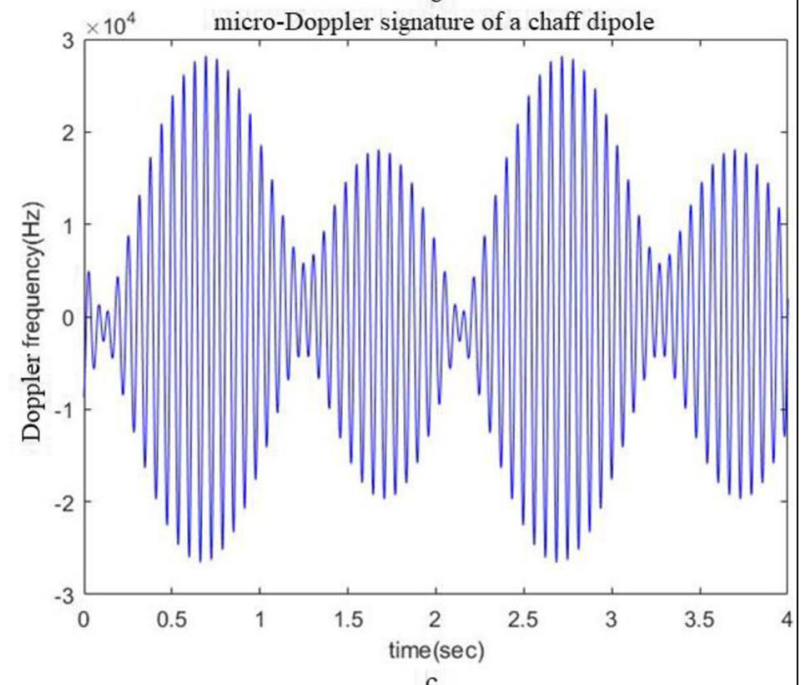

Fig. 6 Micro-Doppler frequency of single chaff dipoles with different azimuths (in the reference coordinates): $\mathbf{a} \theta=30^{\circ}, \varphi=60^{\circ}, d_{r}=722 \mathrm{~m}$, b $\theta=30^{\circ}, \varphi=120^{\circ}, d_{r}=722 \mathrm{~m}$, and $\mathbf{c} \theta=30^{\circ}, \varphi=180^{\circ}, d_{r}=722 \mathrm{~m}$ angular velocity is a counterclockwise precession of the chaff dipole, the bandwidth of the micro-Doppler frequency is positive; otherwise, a negative bandwidth indicates a clockwise precession of the chaff dipole.

Figure $6 \mathrm{a}-\mathrm{c}$ shows the three scattering points selected randomly in the chaff cloud have the same distance $d_{r}$ (in the radar coordinate system), the same elevation $\theta$, and different azimuths $\phi$ (in the reference coordinate system). In contrast, from Fig. $6 a-c$, we can see that the chaff dipoles produce the micro-Doppler frequencies with different bandwidths at different times, and the absolute range of the bandwidth is 15 to $30 \mathrm{kHz}$ through Formula (12). All the periods of the envelopes of the transient bandwidth of the micro-Doppler frequency in different situations are consistent with the angular frequency of the spinning, $f_{\text {envelope }}=0.494 \mathrm{~Hz}$. Ultimately, the changes in the elevation $\theta$ and the azimuth $\phi$ have the same effect on the micro-Doppler frequency of the chaff dipoles.

Figure $7 \mathrm{a}-\mathrm{c}$ shows the three scattering points selected randomly in the chaff cloud have different distances $d_{r}$ (in the radar coordinate system), the same elevation $\theta$, and the same azimuth $\phi$ (in the reference coordinate system). In Fig. 7 , all the periods of the envelopes of the transient bandwidth of the microDoppler frequency in different situations are consistent with the angular frequency of the spinning, $f_{\text {enve- }}$ lope $=0.494 \mathrm{~Hz}$. However, in contrast, in Fig. $7 \mathrm{a}-\mathrm{c}$, we can see that the period change of the micro-Doppler frequency of the chaff dipoles is faster when the range of the transient bandwidth of the microDoppler frequency is smaller. The reason for this phenomenon is that the distances between the chaff dipoles and radar increase, so we can conclude that the change in distance greatly affects the microDoppler frequency.

By comparing Figs. 5, 6, and 7, the following conclusions can be obtained: (1) because each chaff dipole spins at the same constant angular velocity, the envelope of the transient bandwidth of the microDoppler frequency generated by the chaff dipoles has the same modulation period regardless of whether the distances, the azimuths, and the elevation angles are the same or not; (2) when both of the chaff dipoles and the radar system are in the same line (i.e., have the same azimuth angles $\beta$ and the same elevation angles $\alpha$ ), the period change of the micro-Doppler frequency of the chaff dipoles increases with the distances between the chaff dipoles and the radar system increasing, while the range of the transient bandwidth of the micro-Doppler frequency decreases. The farther the chaff dipole is from the center of the radar system, the more remarkable the chaff dipole micromotion effect is. 


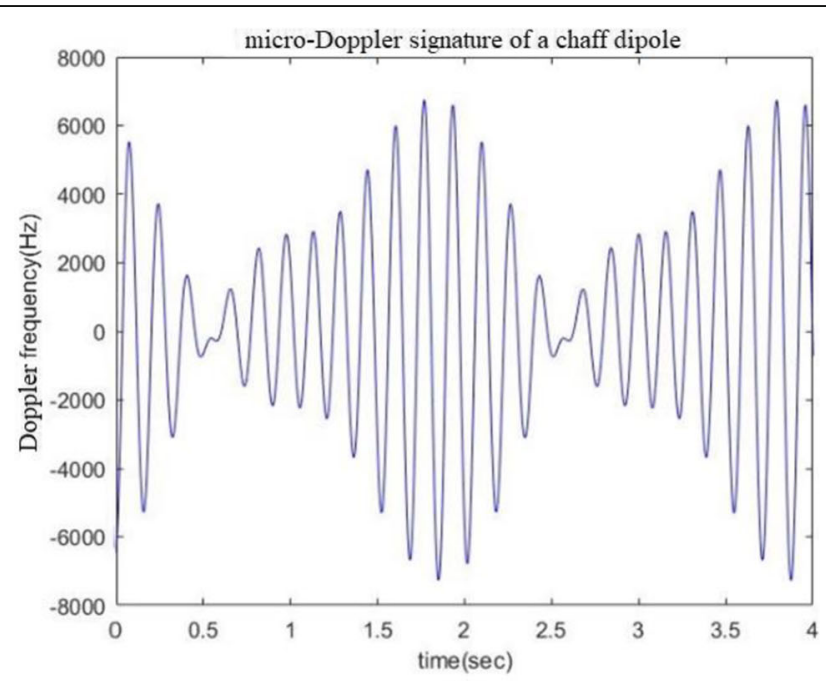

a

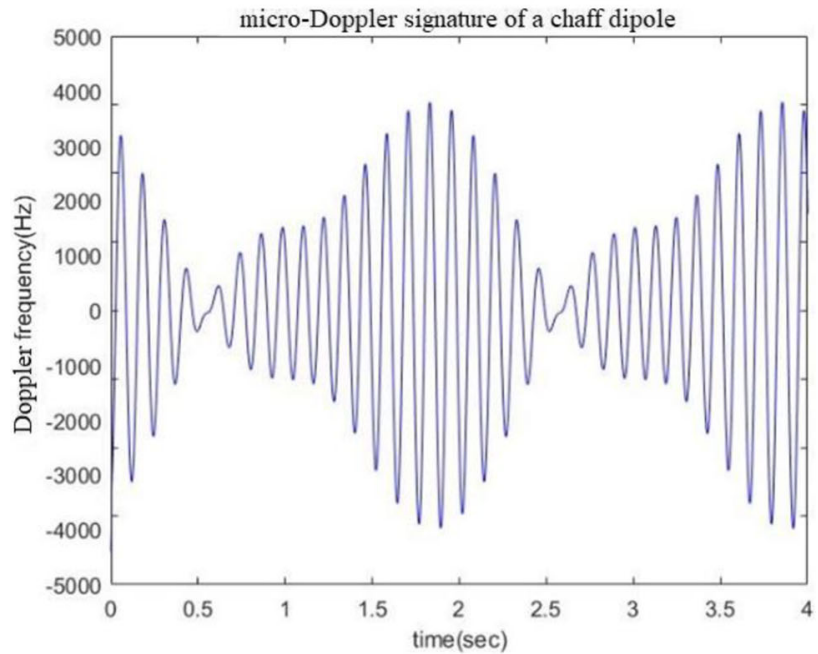

$\mathrm{b}$

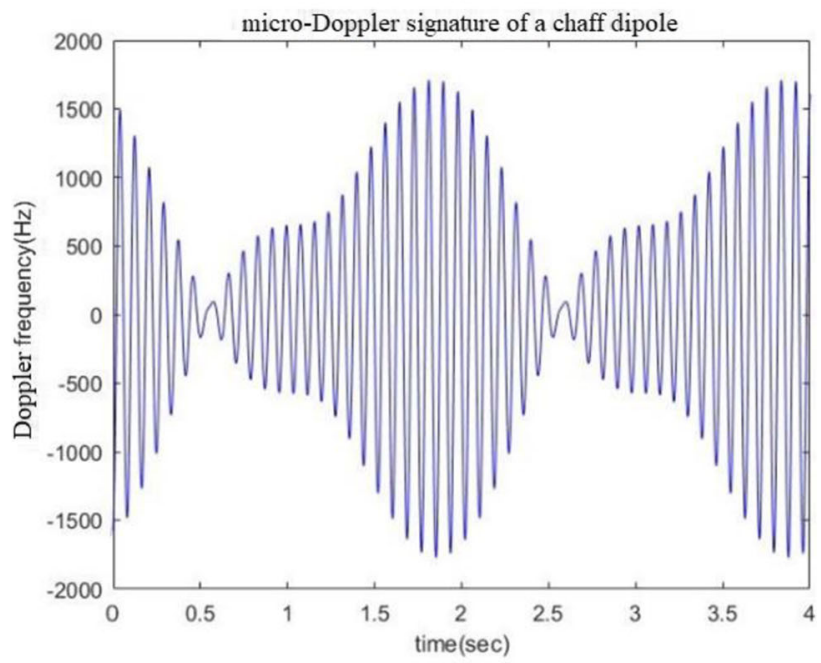

c

Fig. 7 Micro-Doppler frequency of single chaff dipoles with different ranges (in the radar coordinate system): $\mathbf{a} \theta=30^{\circ}, \varphi=30^{\circ}, d_{r}=706 \mathrm{~m}, \mathbf{b} \theta=$ $30^{\circ}, \varphi=30^{\circ}, d_{r}=709 \mathrm{~m}$, and $\mathbf{c} \theta=30^{\circ}, \varphi=30^{\circ}, d_{r}=716 \mathrm{~m}$ 


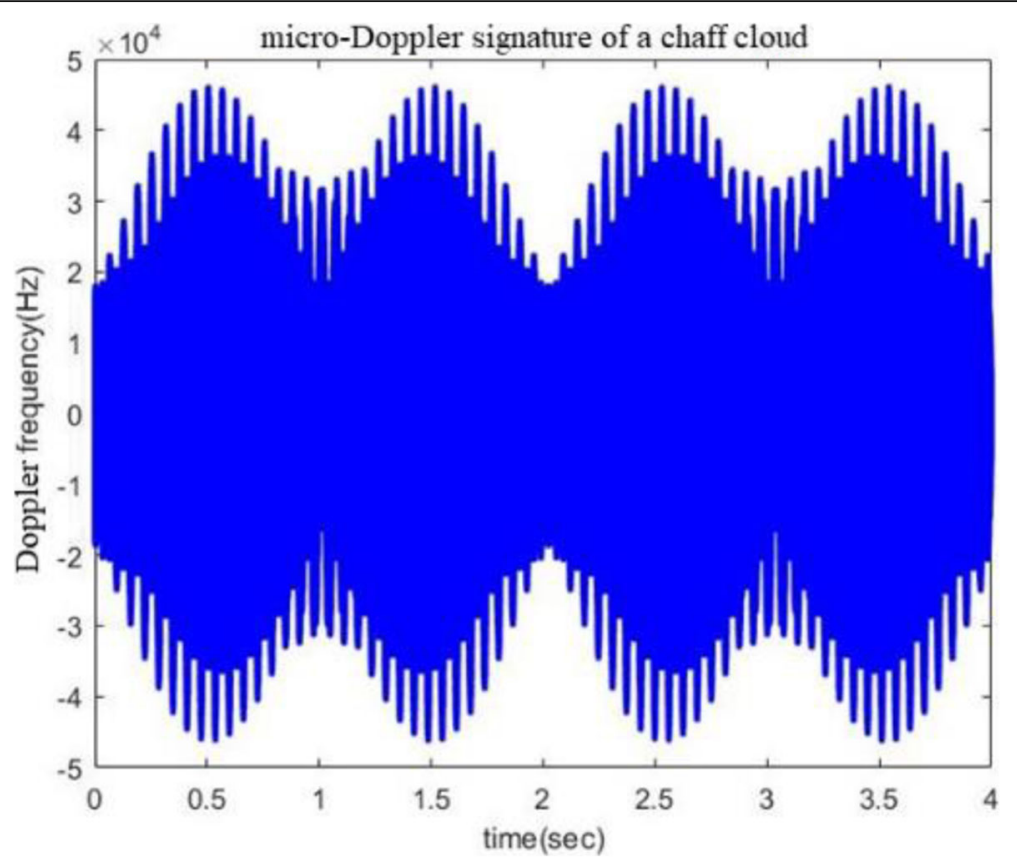

Fig. 8 Time-frequency spectrum of the micro-Doppler frequency of a chaff cloud

Based on the method of multipoint synthesis, it is known that the micro-Doppler spectrum of the chaff cloud echo signal is a set of micro-Doppler frequencies of echoes from all chaff dipoles. Thus, the microDoppler frequency of the chaff cloud is obtained by the micro-Doppler frequencies of the chaff dipoles, which can be expressed as

$$
\exp \left(j 2 \pi f_{C_{-} \text {micro-D }} t\right)=\exp \sum_{k=1}^{K}\left(j 2 \pi f_{k \_ \text {micro-D }} t+\theta_{k}\right)
$$

The parameters of Formula (13) are as follows:

1. $f_{k_{-} \text {micro - } \mathrm{D}-\text { the micro-Doppler frequency of the } k \text { th }}$ chaff dipole.

2. $\theta_{k}$-the initial phase of the $k$ th chaff dipole echo.

3. $K$ is the number of chaff dipoles.

Figure 8 shows the micro-Doppler spectrum of the chaff cloud. From the figure, we can see that the micro-Doppler frequency of the chaff cloud varies periodically like a single chaff dipole, and the maximum absolute value of the bandwidth of the microDoppler frequency of the chaff cloud is $46.1 \mathrm{kHz}$. Therefore, we can draw a conclusion that chaff clouds have periodical variation of micro-Doppler frequencies, and the period is decided by the vector sum of $f_{k_{-} \text {micro - D }}(k=1, \cdots, K)$.
The complex envelope (assumed to be $\theta_{k}=0$ ) of the chaff cloud echoes based on multipoint synthesis can be expressed as:

$$
z(t)=\sum_{k=1}^{K} a_{k} \exp \left(j 2 \pi f_{k \_ \text {micro-D }} t\right)
$$

The parameters of Formula (14) are as follows: $a_{k}-a_{k}=\frac{1}{2} U_{k_{-}} U_{k_{-} r}=\sqrt{\sigma_{k}} / R_{k}^{2} \cdot \varepsilon$.

$U_{k_{-}} t$ the amplitude of the transmitting signal $U_{k_{-} r}$-the amplitude of the echo signal $\sigma_{k}-$ the RCS of the $k$ th chaff dipole

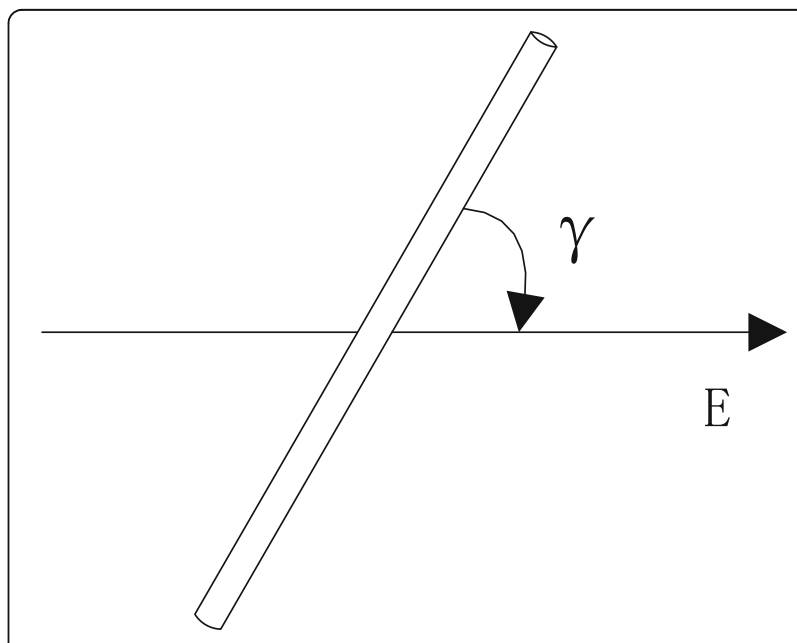

Fig. 9 The resonance angle $\gamma$ 
$R_{k}$-the distance between the $k$ th chaff dipole and the radar system

$\varepsilon-\varepsilon=\frac{1}{2} U_{k \_t} \cdot F_{t}(\Theta) F_{r}(\Theta) \cdot \lambda \sqrt{P_{t} D_{t} D_{r} R_{\Sigma}} / 4 \pi \sqrt{\pi}$

$P_{t}$-the parameters are the power of the transmitting signal

$D_{t}$-the directivity coefficient of the transmitting antenna

$D_{r}$-the receiving antenna

$F_{t}(\Theta)$-the direction function of the transmitting antenna

$F_{r}(\Theta)$-the receiving antenna
According to the induction scattering theory, the effective RCS $\bar{\sigma}$ of a chaff dipole can be expressed as [3]:

$$
\bar{\sigma}=0.86 \lambda^{2} \cos ^{4} \gamma
$$

where $\gamma$ is the angle between the electric field direction of the radar signal and the axis of the chaff dipole, as shown in Fig. 9.

After Formula (15) is substituted into Formula (14), the energy spectrum of the micro-Doppler frequency of the chaff cloud is calculated and simulated by time-
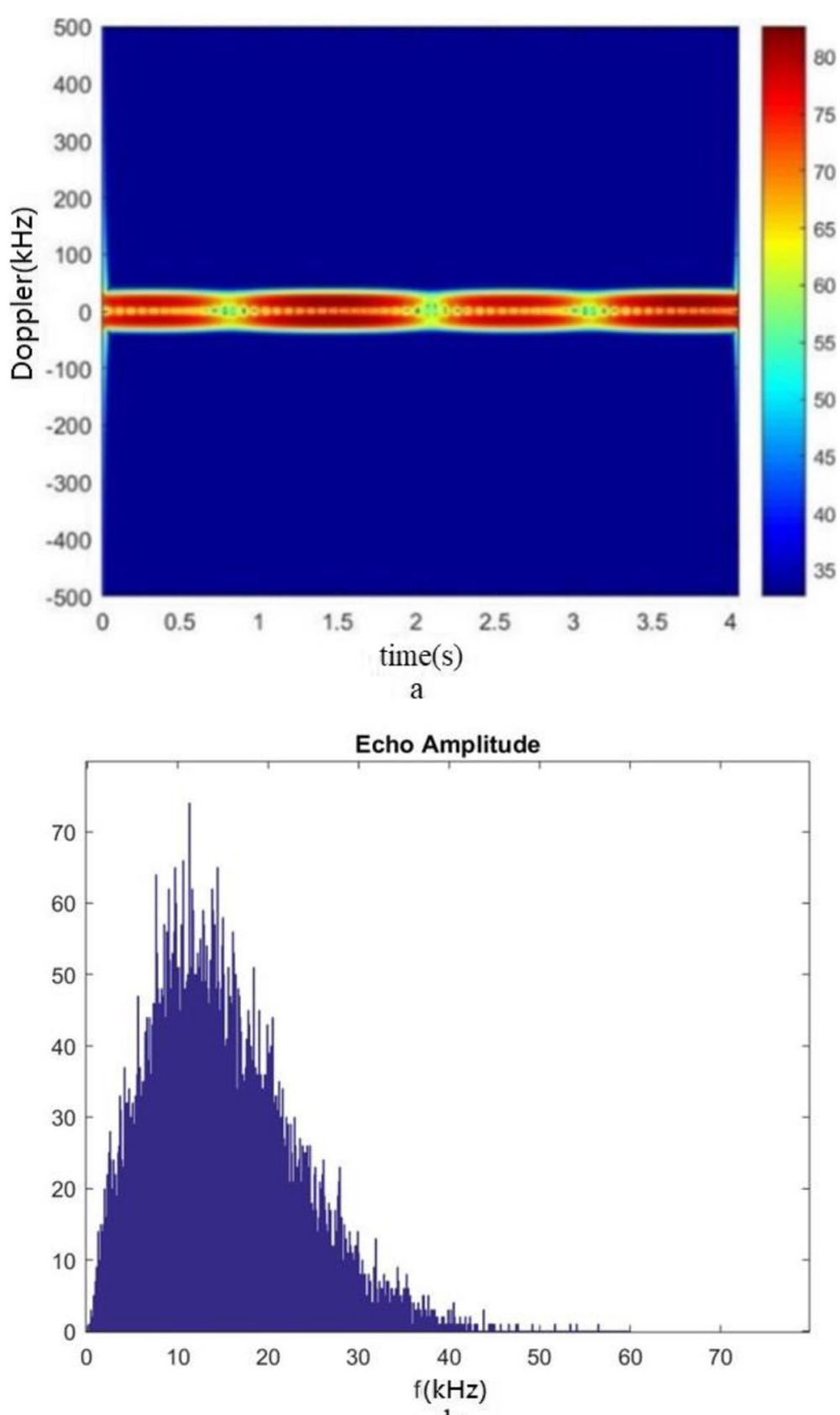

$\mathrm{b}$

Fig. 10 Micro-Doppler spectrum of the chaff cloud: a the energy spectrum of the chaff cloud and $\mathbf{b}$ the echo amplitude of the chaff cloud 
frequency analysis. Figure 10a shows the normalized energy spectrum of the micro-Doppler frequency of the chaff cloud. In Fig. 10b, the amplitude of the micro-Doppler frequency satisfies the Rayleigh distribution, which coincides with the conclusion in the reference [22]. The microDoppler spectrum is further analyzed. The pulse repetition period of pulsed radar determines the sampling rate. When the maximum value of the micro-Doppler frequency is $46.1 \mathrm{kHz}$, the minimum sampling rate is $92.2 \mathrm{kHz}$ and the pulse repetition period is $10 \mu \mathrm{s}$.
Figure 11a shows the micro-Doppler spectrum with a 4096 points/s sampling rate. The periodicity of the chaff cloud micro-Doppler frequency begins to appear, but it is incomplete; Fig. 11b shows the result with a 10,240 points/s sampling rate.

\section{Experimental results}

In this section, experimental trials are conducted to investigate and calculate the micro-Doppler features

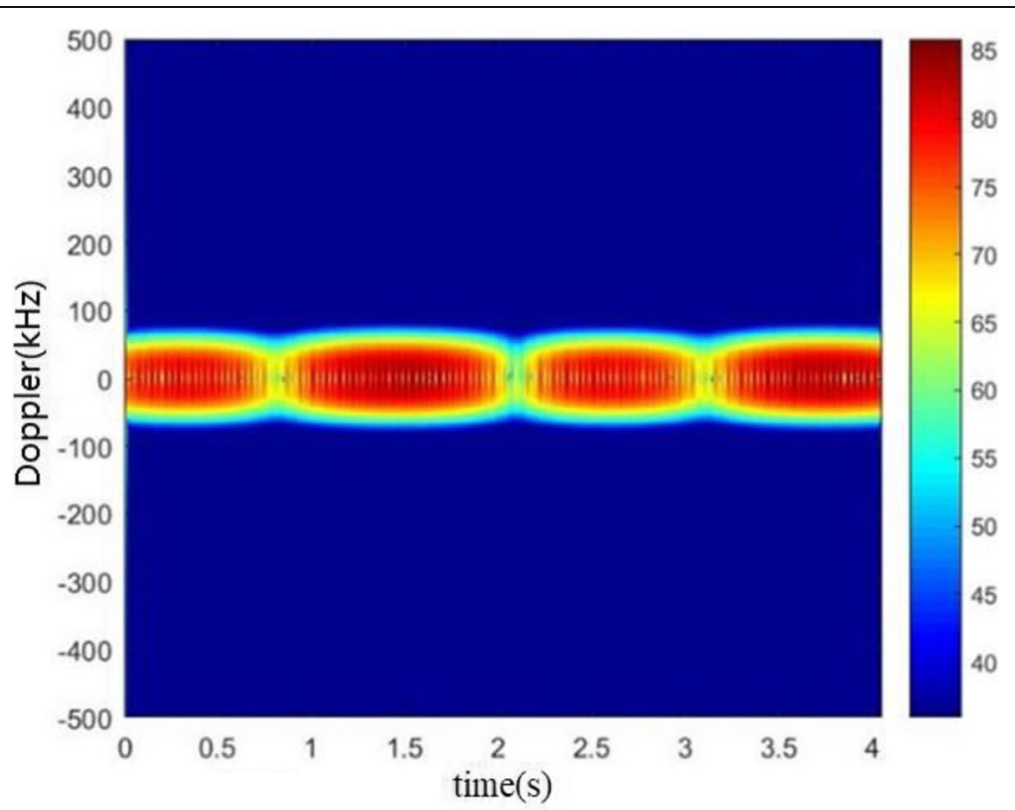

a

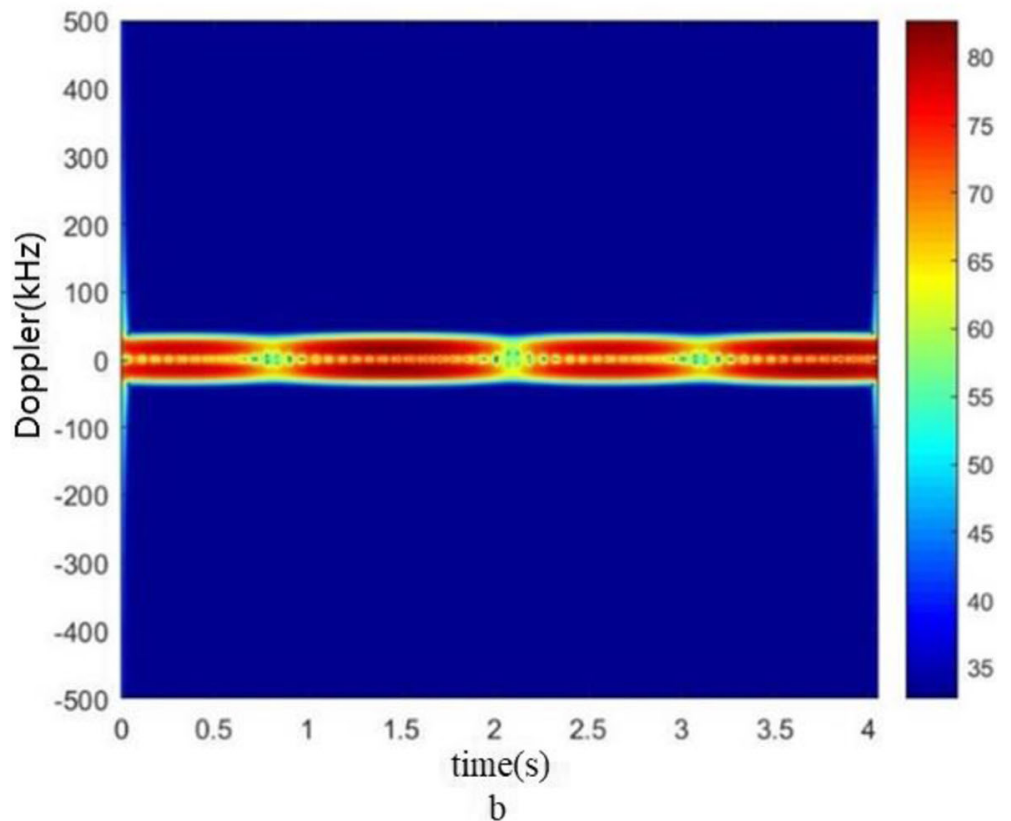

Fig. 11 Micro-Doppler spectrum of the chaff cloud with different sampling rates: a 4096 points/s sampling rate and b 10240 points/s sampling rate 
of a chaff cloud using a C-band transmitted signal. Then, the data collection is performed.

The balloon employed in the experiment throws chaff dipoles above the ground at a height of $1.5 \mathrm{~m}$ through a bracket at a range of $2.5 \mathrm{~m}$ from the transmitting and receiving antennas. The measurement system for this experiment includes a signal generator, data logger, host computer, a transmitting antenna, and a receiving antenna. The experimental procedure is as follows: a balloon full of the chaff dipoles is punctured with a bamboo arrow so that the chaff dipoles are dispersed into the air. Figure 12 shows the balloon throwing device. To accurately measure the chaff dipole echo, the transmitting antenna (horn antenna) and the receiving antenna (horn antenna) are placed in parallel with the chaff cloud, and the antennas are separated by absorbing materials. In this scenario, the signal generator can generate an arbitrary frequency (C-band in this experiment) and an arbitrary waveform signal (linear frequency modulation (LFM) pulse in this experiment). The measurement system receives an echo signal from the receiving antenna when the signal is transmitted from the signal generator at the same time. Then, the system processes and records the experimental data. By using a host computer that can monitor the entire test system, the data can be analyzed, and the experimental results can be shown in a time-frequency diagram.

According to the experimental results, the data that include the movement characteristics of the chaff dipoles and chaff cloud are selected for analysis. To conveniently describe the development of chaff clouds, the instantaneous time of the bamboo arrow piercing the balloon is set to time 0 . The experimental image in Fig. 12 shows that approximately $20 \mathrm{~g}$ of chaff dipoles passed through several stages after the balloon burst, as the emergence of the chaff dipoles from the balloon, chaff dipoles separation, and chaff cloud deposition.

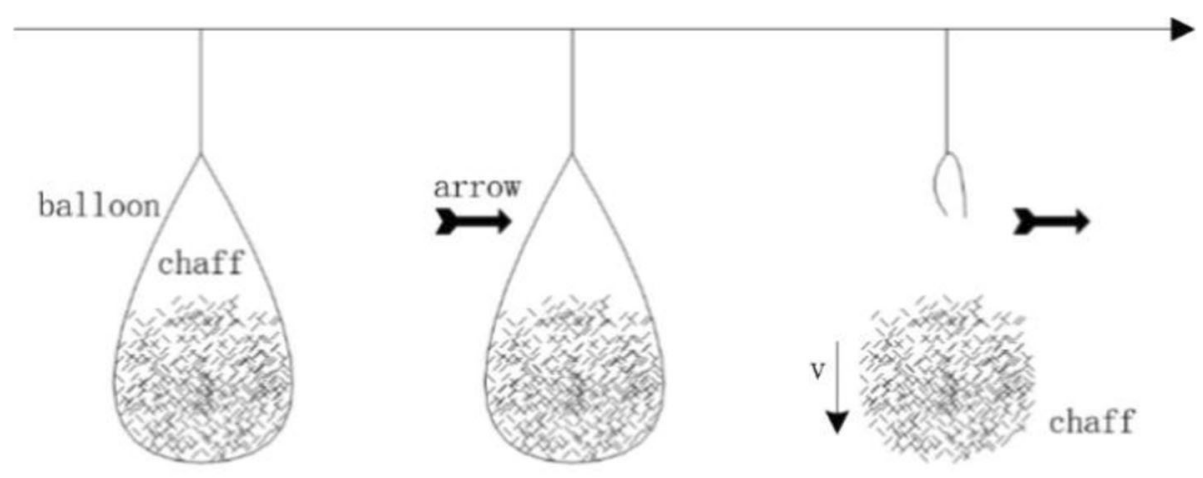

a

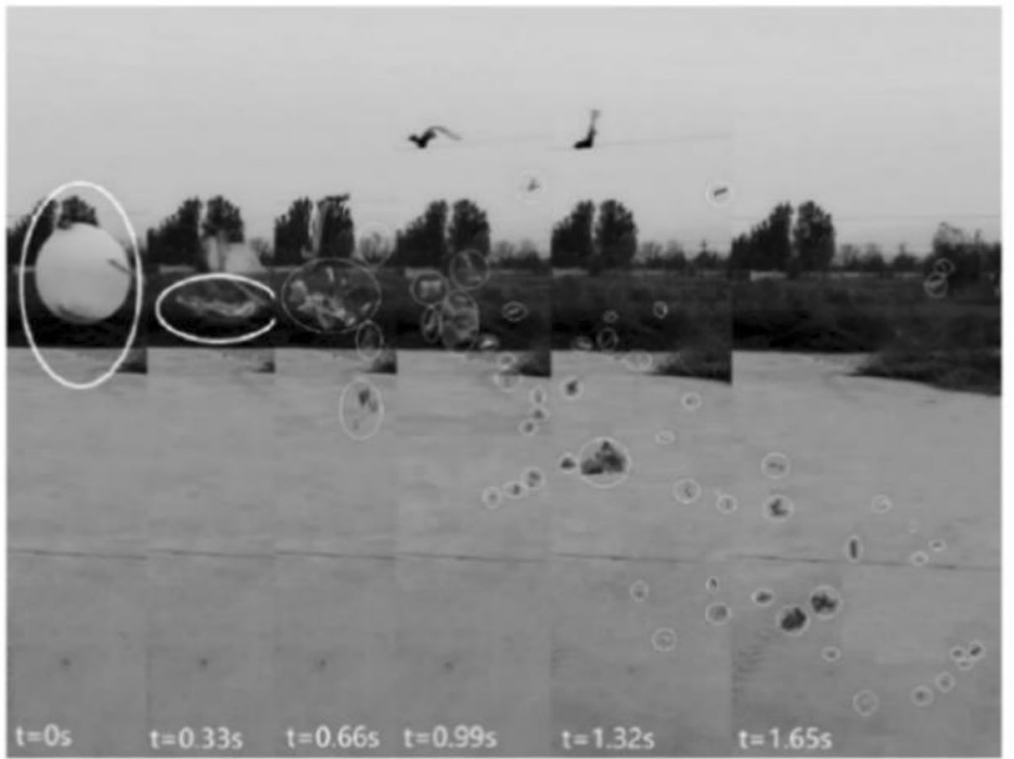

b

Fig. 12 Chaff dipole dispersion experiment: a chaff dipoles thrown from a balloon, and $\mathbf{b}$ the chaff dipole dispersion distribution 
To demonstrate the procedure of micro-Doppler analysis, the results of the trial are presented. First, a Fourier transform of the original radar returned data is computed, and the obtained image is shown in Fig. 13. As seen from the image, a main frequency bin with a large amplitude exists in the middle of the spectrum representing the chaff cloud radial motion. Surrounding this frequency bin, one can observe other side peaks representing the frequency of the chaff dipoles. The micro-Doppler features are extracted by using the EMD method as described below.

The EMD algorithm consists of two parts. The first part is the intrinsic-mode functions (IMFs), which decomposes the real-valued signal into a finite number of IMFs. The second part is the calculation of the Hilbert spectrum, in which the analytical signal for each IMF is obtained via Hilbert transform. Then, the distribution of energy-frequency-time can be reconstructed from the original signal. Figure 14 shows the data analysis procedure. First, all the local extremum points of the signal $x(t)$ are determined. Next, the local maximum upper envelope $p(\mathrm{t})$ and the local minimum lower envelope $q(\mathrm{t})$ are fitted by a cubic spline difference. The mean of the upper and lower envelope curve $m(t)=(p(t)+q(t)) / 2$ is taken as the mean envelope of the signal $x(\mathrm{t})$. Second, the highfrequency sequence $\mathrm{h}(\mathrm{t})$ is obtained by subtracting the mean $m(\mathrm{t})$ from $x(\mathrm{t}), h(t)=x(t)-m(t)$. According to the above rules, the steps are repeated until the stopping condition of the sifting IMF component is satisfied, $\mathrm{SD} \in(0.2,0.3)$ [23]. Then, the first IMF component $c_{1}(t)$ is obtained. Finally, the new signal $r_{1}(t)$ is obtained by subtracting $c_{1}(t)$ from $x(\mathrm{t})$, and the process is repeated until the last $r_{n}(t)$ cannot be decomposed so that all IMFs can be obtained. After the EMD of the original signal $x(\mathrm{t})$, the IMFs $c_{i}(t)(i \in$ $(1,2, \ldots, n))$ with a frequency range from high to low and the remainder $r_{n}(t)$ are obtained.

Thus, the original signal can be expressed as a sum of IMFs and the remainder.

$$
x(t)=\sum_{i=1}^{n} c_{i}(t)+r_{n}(t)
$$

where the threshold is defined as [23].

$$
S D=\sum_{t=0}^{T}\left[\frac{\left|h_{i-1}(t)-h_{i}(t)\right|}{h_{i}^{2}(t)}\right]
$$

The subscript denotes the $i$ th sifting process.

Figure 15 shows the EMD results. In this figure, the micro-Doppler spectrum is decomposed into a series of IMFs. In the IMF data, imf 1 mainly consists of a high-frequency signal. Meanwhile, the signals of imf $2 \sim \operatorname{imf} 5$ have the largest energy, and the signal amplitude varies dramatically. The remaining $\operatorname{imf} 6, \operatorname{imf} 7$, and res mainly consist of low-frequency signals.

The next step in the procedure is to employ timefrequency analysis to depict the micro-Doppler changes and to estimate the motion parameters of the chaff dipoles. The time-frequency feature of the EMD

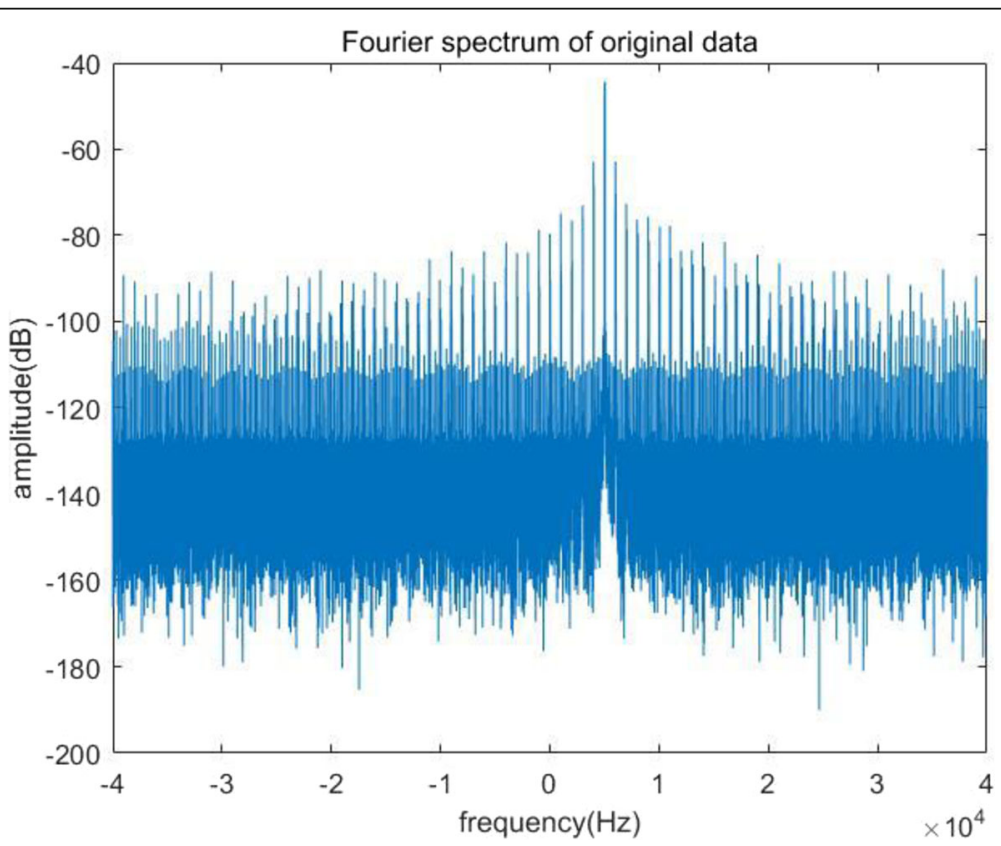

Fig. 13 Spectrum of the original signal echo 


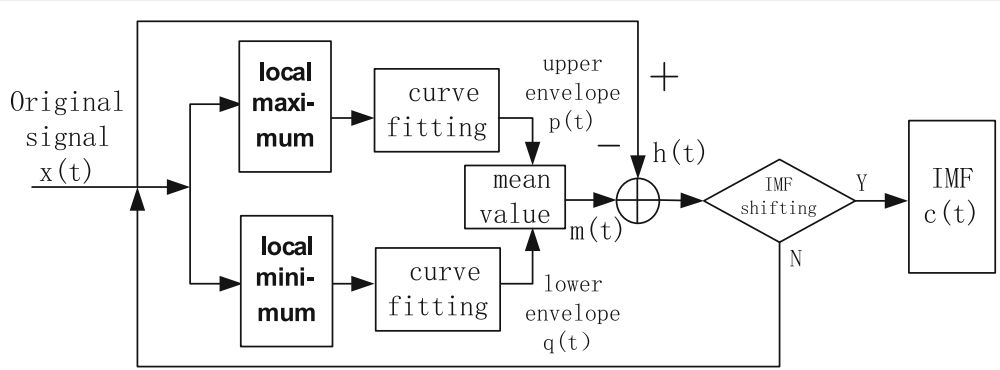

Fig. 14 EMD flowchart

signals obtained by using the STFT is given in Fig. 16 . The chaff dipoles are observed as the periodic variation at $0 \mathrm{~Hz}$ on the frequency axis by filtering the chaff cloud Doppler frequency, which is caused by radial motion in radar using the EMD method. Note that image intensity scales are normalized. The period of the strong flashes is the precession period of unexpanded chaff dipole cluster. By comparing Fig. 16 and Fig. 10, we can see that the theoretical data and experimental data have similar micromotion features. In Fig. 16, the energy of the chaff cloud satisfies the Rayleigh distribution, and the micro-Doppler frequency of the chaff cloud varies periodically. The difference is caused by the incompletely expanded chaff dipoles in the experiment.

\section{Conclusion}

In this paper, we obtained a precession model of chaff dipoles and a steady-state model of a chaff cloud. In these models, the position model of a single chaff dipole is deduced by the Euler coordinate transformation; the precession model is given by the dynamic characteristics of the chaff dipole. Furthermore, based on the theory of the micromotion characteristics of a single chaff dipole, the micro-Doppler frequency characteristics of a chaff cloud are quantified by multipoint synthesis method. Finally, a numerical solution of the micro-Doppler frequency of the chaff cloud is shown as a time-frequency spectrum of the chaff cloud. Through a quantitative analysis of the micro-Doppler frequency characteristics of the chaff cloud, the following conclusions can be drawn. (1) Because each chaff dipole moves precessionally at the same constant angular velocity, the envelope of the transient bandwidth of the micro-Doppler frequency generated by the chaff dipoles has the same modulation period regardless of whether the distances, the azimuths, and the elevation angles are the same or not. (2) When both of the chaff dipoles and the radar system are in the

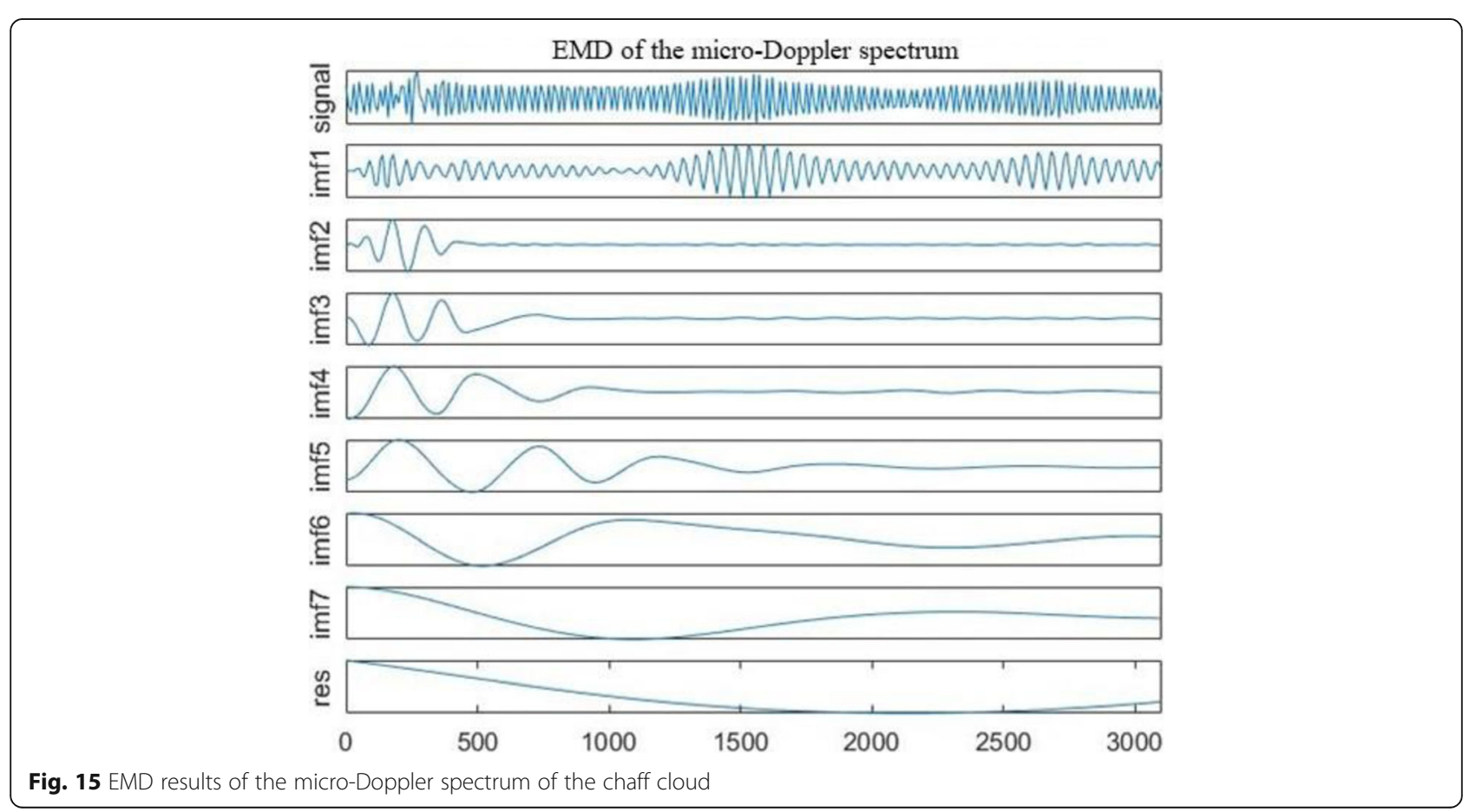




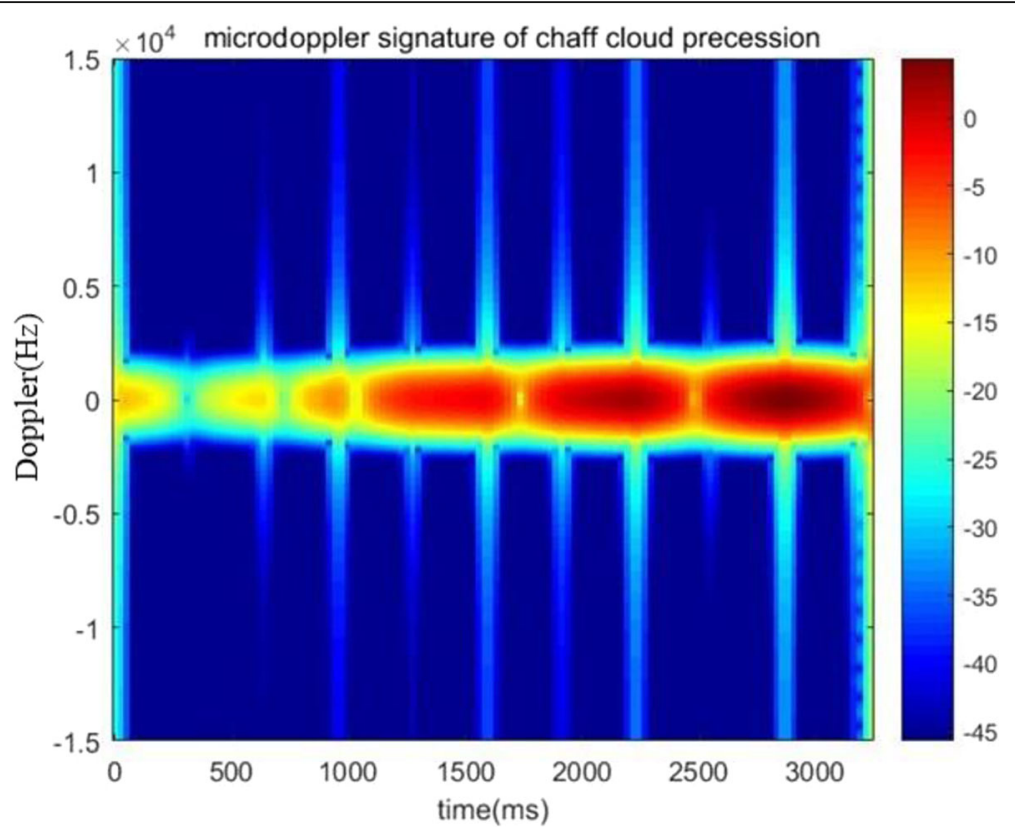

Fig. 16 Time-frequency signatures of the precession from the chaff cloud

same line (i.e., have the same azimuth angles $\beta$ and the same elevation angles $\alpha$ ), the period change of the micro-Doppler frequency of the chaff dipoles increases with the distances between the chaff dipoles and the radar system increasing, while the range of the transient bandwidth of the micro-Doppler frequency decreases. (3) Chaff clouds have periodically varying micro-Doppler frequencies, and this period is determined by the vector sum of the micro-Doppler frequencies of all the chaff dipoles; (4) the amplitude of the micro-Doppler frequencies of the chaff cloud satisfies the Rayleigh distribution.

Through the micromotion effect, the characteristics of the micro-Doppler frequencies can be used as an effective feature to recognize a chaff cloud and targets (such aircraft). In the following work, the micromotion classification technologies (e.g., [12-14]) may be combined with the micromotion characteristics of the chaff cloud, to effectively classify chaff cloud and targets.

\section{Abbreviations}

CW: Continuous-wave; EMD: Empirical mode decomposition;

HCA: Hydrometeor classification algorithm; IMFS: Intrinsic-mode functions;

LFM: Linear frequency modulation; RCS: Radar cross section; STFT: Short-time Fourier transform; SVM: Support vector machine

\section{Acknowledgements}

Authors have obtained permission to acknowledge anyone who contributed towards the article who does not meet the criteria for authorship including anyone who provided professional writing services or materials.

\section{Authors' contributions}

$R L$ wrote the draft. $R L, X H$, and $P L$ did the experiment. $X H$ and $P L$ reviewed and revised the draft. All authors read and approved the final manuscript.

\section{Authors' information}

Xinhong Hao was born in Henan Province, China, in 1974. She received the Ph.D. degree in mechatronic engineering from Beijing Institute of

Technology, in 2007. She is now an Associate Professor in Beijing Institute of Technology. Her main research interests include target detection theory of radio sensor and signal processing, real-time signal processing, and timefrequency analysis.

Ran li received the B.E. degree from North University of China, China, in 2012, the M.E. degree from North University of China, China, in 2015. She is now a PhD student at the School of Mechatronical Engineering, Beijing Institute of Technology, China.

Ping Li received the B.E. degree from Dalian Railway Institute, China, in 1985, the M.E. degree from Dalian Institute of Technology, China, in 1987, and the Ph.D. degree from the School of Mechatronical Engineering, Beijing Institute of Technology, China, in 1995. She is now a Professor at the School of Mechatronical Engineering, Beijing Institute of Technology, China. Her current research interests include proximity detection and signal processing.

\section{Funding}

This work is supported by the nation pre-research foundation for China (grant nos.61406190101).

\section{Availability of data and materials}

These data in the paper are the results of simulations and experiments from the Science and Technology on Electromechanical Dynamic Control

Laboratory. Please contact author for data requests.

\section{Consent for publication}

All presentations of case reports must have consent to publish.

\section{Competing interests}

The authors declare that they have no competing interests.

Received: 10 June 2019 Accepted: 27 September 2019

Published online: 01 November 2019

\section{References}

1. B.C.F. Butters, At 'chaff', communication, radar and signal processing. IEEE Proc. F 129(3), 197-201 (1982)

2. J. Chen. Principles of radar chaff jamming. Beijing: National Defense Industry Press, 212-245 (2008) 
3. Y. Liu, S. Xing, Y. Li, et al., Jamming recognition method based on the polarisation scattering characteristics of chaff clouds. IET Radar Sonar Navigation 11(11), 1689-1699 (2017)

4. $\quad V$. Chen, The micro-doppler effect in radar (2011)

5. Deng B, Li X, et al., Theories \& methods for SAR micro-motion target detection and imaging. Beijing: Science Press. 21-32 (2014)

6. Li K L, Research on feature extraction and parameters estimation technology of radar targets with micro-motions. Changsha: National University of Defense Technology. 56-73 (2010)

7. A.R. Persico, C. Clemente, D. Gaglione, et al., On Model, Algorithms and Experiment for Micro-Doppler based Recognition of Ballistic Targets. IEEE Transactions on Aerospace and Electronic Systems. (53):1-1 (2017)

8. A.R. Persico, C.V. llioudis, C. Clemente, et al., Novel classification algorithm for ballistic target based on HRRP frame. IEEE Trans. Aerosp. Electron. Syst., 1-1 (2019) https://doi.org/10.1109/TAES.2019.2905281

9. T. Thayaparan, S. Abrol, E. Riseborough, Analysis of radar micro-Doppler signatures from experimental helicopter and human data. IET Radar Sonar Navigation 1(4), 289-299 (2007)

10. J. Li, H. Ling, Application of adaptive chirplet representation for ISAR feature extraction from targets with rotating parts. IEE Proc Radar Sonar Navigation 150(4), 284-280 (2003)

11. G. Li, P.K. Varshney, Micro-Doppler parameter estimation via parametric sparse representation and pruned orthogonal matching pursuit. IEEE J. Selected Top. Appl. Earth Observ. Remote Sensing 7(12), 4937-4948 (2014)

12. C. Clemente, L. Pallotta, A. Maio, et al., A novel algorithm for radar classification based on doppler characteristics exploiting orthogonal pseudo-Zernike polynomials. IEEE Trans. Aerosp. Electron. Syst. 51(1), 417430 (2015)

13. X. Bai, M. Xing, F. Zhou, et al., Imaging of micromotion targets with rotating parts based on empirical-mode decomposition. IEEE Trans. Geosci. Remote Sens. 46(11), 3514-3523 (2008)

14. Y. Kim, H. Ling, Human activity classification based on micro-Doppler signatures using a support vector machine. IEEE Trans. Geosci. Remote Sensing 47(5), 1328-1337 (2009)

15. J. Brunk, D. Mihora, P. Jaffe, Chaff aerodynamics (1975)

16. Kurdzo J M, Bennett B J, Veillette M S, et al. WSR-88D chaff detection and characterization using an optimized hydrometeor classification algorithm. AMS Conference on Aviation, Range, and Aerospace Meteorology. 2017

17. H. Park, A.V. Ryzhkov, D.S. Zrnić, et al., The hydrometeor classification algorithm for the polarimetric WSR-88D: description and application to an MCS. Weather Forecast. 24(3), 730-748 (2008)

18. Shang W, Chen B X, Jiang L F. An anti-chaff jamming method based on the effect of spectral expansion. Guidance \& Fuze.2006

19. M. Bendayan, A. Garcia, Signal modeling of chaff in naval environment simulation. IEEE Trans Aerospace Electron. Syst. 51(4), 3161-3166 (2015)

20. Xuesong W, Zhijie C, Yongzhen L. Polarization scattering characteristics of chaff cloud in outerspace. 2001 CIE International Conference on Radar Proceedings. Beijing: IEEE. 444-448 (2001)

21. Dong J, Wang F D, Liu Z F. Research into chaff jamming cancellation technology of multiple MTI based on Doppler filter. Shipboard Electronic Countermeasure. 2013

22. N.M. Korah, G. Hua,et al., Principle of radar fuze. Beijing: National Defense Industry Press, 126-137 (1980)

23. X. Bai, M. Xing, F. Zhou, et al., Imaging of micromotion targets with rotating parts based on empirical-mode decomposition. IEEE Trans. Geosci. Remote Sens. 46(11), 35143523 (2008)

\section{Publisher's Note}

Springer Nature remains neutral with regard to jurisdictional claims in published maps and institutional affiliations.

\section{Submit your manuscript to a SpringerOpen ${ }^{\circ}$ journal and benefit from:}

- Convenient online submission

- Rigorous peer review

- Open access: articles freely available online

- High visibility within the field

- Retaining the copyright to your article

Submit your next manuscript at $\boldsymbol{\nabla}$ springeropen.com 